\section{Katze rausgeklagt}

\begin{abstract}
llergiker haben ein Recht auf eine katzenfreie Nachbarschaft, das haben Münchner Richter jetzt bestätigt. Ein Wohnungsinhaber hatte von seinen Nachbarn die Abschaffung ihrer Katze verlangt. Der Mann wies per Attest nach, dass die Katzenhaare bei ihm einen lebensbedrohlichen Asthmaanfall auslösen könnten. Die Katzenbesitzer argumentierten dagegen, dass das Tier nur als Wohnungskatze gehalten werde und zudem wichtig sei für die psychotherapeutische Behandlung ihres 12-jährigen Sohnes, der an Panikattacken und Sprachstörungen leide. Das Amtsgericht München entschied, dass die Vermeidung eines Asthmaanfalls schwerer wiege als die Unterstützung einer psychischen
\end{abstract}

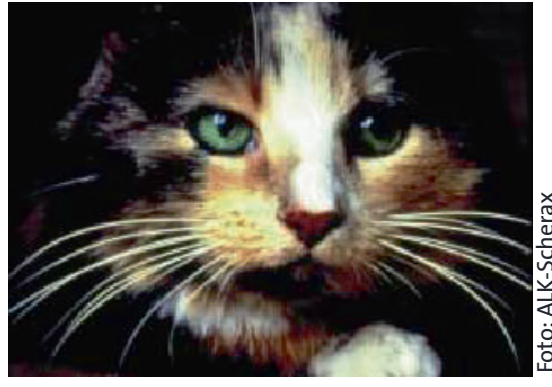

Behandlung. Das Landgericht München schloss sich in der Berufung an: Natürlich werde der Kläger im täglichen Leben immer wieder mal Katzen begegnen, „während er hier jedoch ausweichen kann, besteht diese Möglichkeit im eigenen Wohnbereich gerade nicht".

es

Amtsgericht München, Az. 191 C 10647/03; Landgericht München I, Az. 34 S 16167/03

\title{
Hygiene-Hypothese neu diskutiert
}

nfektionen vor dem 6. Lebensmonat scheinen nicht vor Neurodermitis zu schützen. Dies stellten dänische Wissenschaftler in einer Kohortenstudie mit rund 24.000 Kindern fest. Ganz im Gegenteil: Mit jeder infektiösen Erkrankung stieg das Risiko für ein atopisches Ekzem (Verhältnis der Inzidenzraten 1,08). Bei $85 \%$ der Infektionen handel- te es sich um banale Erkältungen. Hingegen verminderten der Besuch einer Kinderkrippe vor dem 6. Lebensmonat $(0,82)$, drei oder mehr Geschwister $(<0,86)$, Haustiere $(0,87)$ und das Aufwachsen auf einem Bauernhof $(0,90)$ das Risiko für ein atopisches Ekzem. nz

Benn CS et al. BMJ 2004; 328: 1223-6

Alle Jahrgänge des Allergo Journal zurück
bis in das Jahr 1997 online recherchierbar
- das ist das Highlight des neuen Inter-
net-Auftritts www.allergo-journal.de. Das
Online-Archiv wird dabei sowohl durch
eine Volltextsuche als auch eine
Originalia-Suche erschlossen, bei der

\section{Milbensuche in Island}

sland gilt wegen seiner Abgeschiedenheit und seines rauen Klimas als hausstaubmilbenfreie Zone. Umso überraschter war deshalb ein skandinavisches Ärzteteam, das bei 9\% der jungen erwachsenen Bevölkerung der Hauptstadt Reykjavik spezifisches IgE gegen Dermatophagoides pteronyssinus dokumentieren konnte. Eine sofort begonnene Milbensuche in 197 Wohnungen erbrachte insgesamt lediglich zwei Exemplare von D. pteronyssinus, eines davon bereits tot. Der p 1 ließ sich in keiner der gewonnenen Staubproben nachweisen. Als Sensibilisierungsweg vermuten die Forscher jetzt eine Allergenexposition auf Reisen oder durch von Zugvögeln mitgebrachte Milben. Auch eine Kreuzreaktivität mit anderen Milbenspezies könnte möglich sein. $n w$

Hallas TE et al. Allergy 2004; 59:515-9

\section{Maritime Allergene}

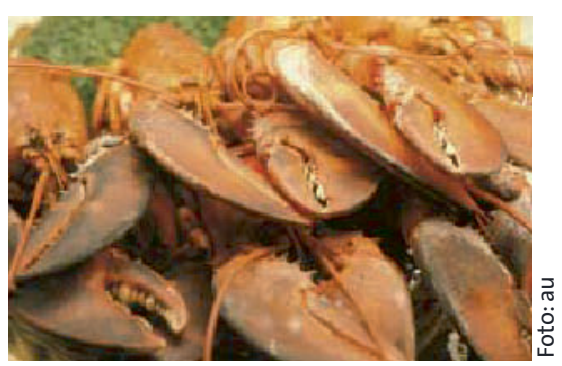

A llergien gegen Fische und Krustentiere nehmen zu. Eine Studie der Mount Sinai School of Medicine stellte fest, dass derzeit bereits einer von 50 US-Amerikanern auf Schalentiere und einer von 250 auf Fische allergisch reagiert. Das Besondere dabei: Bei rund der Hälfte der Fälle beginnt die Allergie erst im Erwachsenenalter und schwere Reaktionen sind häufiger als bei anderen Formen der Nahrungsmittelallergie. Als Ursache für die Entwicklung vermuten die Autoren unter anderem veränderte Ernährungsgewohnheiten.

$D E$

Sicherer SH et al. J Allergy Clin Immunol 2004; 114: 159-65 